Article

\title{
New Multiple Attribute Decision Making Method Based on DEMATEL and TOPSIS for Multi-Valued Interval Neutrosophic Sets
}

\author{
Wei Yang * and Yongfeng Pang \\ Department of Mathematics, School of Science, Xi'an University of Architecture and Technology, \\ Xi'an 710055, China; pangyongfeng75@163.com \\ * Correspondence: yangweipyf@163.com
}

Received: 20 March 2018; Accepted: 10 April 2018; Published: 17 April 2018

\begin{abstract}
Interval neutrosophic fuzzy decision making is an important part of decision making under uncertainty, which is based on preference order. In this study, a new multi-valued interval neutrosophic fuzzy multiple attribute decision making method has been developed by integrating the DEMATEL (decision making trial and evaluation laboratory) method and the TOPSIS (the technique for order preference by similarity to an ideal solution) method. Evaluation values are given in the form of multi-valued interval neutrosophic fuzzy values. By using DEMATEL, dependencies among attributes can be modeled, and attribute weights are determined. The normalized Euclidean distance and the normalized Hamming distance between multi-valued interval neutrosophic fuzzy values are defined. Alternatives are ranked by using the TOPSIS method based on the distance measures. The truck selection problem in the logistics industry is presented to illustrate the feasibility and practical advantages of the proposed method. Some comparisons of the new method with other methods are also made.
\end{abstract}

Keywords: multiple attribute decision making; multi-valued interval neutrosophic set; DEMATEL; distance measure; TOPSIS; aggregation operator

\section{Introduction}

Decision making problems have become more complicated because of the increasing amount of decision information and alternatives, the inherent uncertainty and complexity of decision problems, the fuzzy nature of human thinking, etc. Many useful tools have been developed to model fuzzy and uncertain information. The neutrosophic set, introduced by Smarandache [1], is a powerful extension of some classic sets including the fuzzy set [2], the interval-valued fuzzy set [3], the intuitionistic fuzzy set [4], the hesitant fuzzy set [5], etc. In the neutrosophic set, each element has a truth-membership, an indeterminacy-membership and a falsity-membership belonging to the real standard or non-standard subset of $] 0^{-}, 1^{+}$. Compared to other tools to model fuzzy, inconsistent and uncertain information, the neutrosophic set is more flexible and accurate, which is based on preference order. The neutrosophic set has received broad attention and has been applied extensively [6-12]. Wang et al. [13] proposed a single-valued neutrosophic set and studied various properties of the single-valued neutrosophic set. Ye studied the correlation coefficient of the single-valued neutrosophic set in [14] and presented the cross-entropy single-valued neutrosophic set in [15]. Yang et al. [16] studied the single-valued neutrosophic relations. TOPSIS in the single-valued neutrosophic environment has been studied by Biswas et al. [17]. Similarity and entropy in the neutrosophic environment were studied by Majumdar and Samant [18]. Liu et al. [19] presented some generalized neutrosophic Hamacher aggregation operators. Wang and Li [20] developed a 
multi-valued neutrosophic set and presented the TODIMmethod based on multi-valued neutrosophic fuzzy information. Researchers further developed neutrosophic soft sets [21], trapezoidal neutrosophic sets [22], single-valued neutrosophic linguistic sets [23], etc. In the evaluation process, decision makers would like to evaluate with interval numbers rather than crisp numbers due to the fuzzy nature of human thinking. Wang et al. [24] developed the set-theoretic operators on the neutrosophic set called the interval neutrosophic set. Rdvan [25] developed some cross-entropy measures on interval neutrosophic fuzzy sets. Liu and Tang [26] proposed some power generalized aggregation operators for interval neutrosophic information. Liu and Shi [27] presented some generalized hybrid weighted averaging operators for interval neutrosophic hesitant fuzzy information. Zhang et al. developed an outranking method for interval neutrosophic sets. Yang et al. [28] proposed the linear assignment method for interval neutrosophic sets. Chi and Liu [29] extended the TOPSIS method to the interval neutrosophic environment. In complicated decision problems, several experts from different fields have been invited to evaluate alternatives with respect to the attributes, and these experts can provide several evaluation values for hesitation in decision making or can refuse to give any evaluation values if they are unfamiliar with the attributes. Then, we can obtain multi-valued interval neutrosophic values. Though several interval neutrosophic decision methods have been developed, most of them are based on single-valued interval neutrosophic values, and the problems with multi-valued interval neutrosophic values have rarely been studied. In order to deal with complicated decision problems with multiple decision makers involved, the evaluation values of decision makers are given in the form of multi-valued interval neutrosophic values in this paper.

DEMATEL was first developed by the Science and Human affairs Program of the Battelle Memorial Institute of Geneva [30]. Stević et al. [31] used DEMATEL and the analytic network process (D-ANP) to determine the weights of factors on the S\&P 500 Index futures. The DEMATEL method can be used to identify the possible interdependence among the elements by using the structural modeling technique. The DEMATEL method has been studied extensively and applied in a broad range of fields. Lin and $\mathrm{Wu}$ [32] develop a fuzzy DEMATEL method, in which the evaluation values are triangular fuzzy numbers. Jassbi et al. [33] built a strategy map by using fuzzy DEMATEL. The DEMATEL method in the intuitionistic fuzzy environment has been studied by Nikjoo and Saeedpoor in [34]. Govindan in [35] applied the intuitionistic fuzzy DEMATEL to green supply chain management. DEMATEL for the type-2 fuzzy set has been presented by Hosseini and Tarokh [36]. Abdullah and Zulkifli [37] further extended DEMATEL to develop interval type-2 fuzzy DEMATEL, in which fuzzy AHPand interval type-2 fuzzy DEMATEL have been integrated. DEMATEL has been applied in supply chain management, hospital service, emergency management, investment portfolio projects, etc. DEMATEL has been integrated with other decision making methods to solve decision problems, including the ANP, AHP, TOPSIS, VIKOR, grey analysis, ELECTRE, etc. Though several methods based on DEMATEL have been developed, the study of DEMATEL in the multi-valued interval neutrosophic environment has yet to be found. How to determine attribute weights is very important in the decision making process since reasonable weights can ensure scientific and reasonable decision results. DEMATEL can be used to analyze the complex relationship among attributes and their model dependencies. In this paper, the DEMATEL method has been used to determine attribute weights in the multi-valued interval neutrosophic environment. The TOPSIS method was developed by Hwang and Yong [38], which is based on the idea that the optimal alternative should be the one that is closest to the positive ideal solution and farthest from the negative ideal solution at the same time. TOPSIS has been successfully applied in a wide variety of decision problems due to its comprehensibility and simplicity [39-42]. In TOPSIS, how to determine reasonable attribute weights is very important, which could affect whether the decision results are reasonable and efficient. The DEMATEL method can model the relations among criteria by considering the causes and effects of criteria. Thus, more effective attribute weights can be obtained by using the DEMATEL method compared to the existing attribute methods, such as subjective weights or equal weights. In this paper, we integrate the DEMATEL method with the TOPSIS method for multi-valued interval neutrosophic information. The evaluation values are in the 
form of multi-valued interval neutrosophic values, which can more accurately model the uncertainty, hesitation and fuzziness that exist in the decision making process. The DEMATEL method has been used to determine attribute weights, which can model the dependency among attributes. We define the multi-valued interval neutrosophic fuzzy normalized Euclidean distance and multi-valued interval neutrosophic fuzzy normalized Hamming distance, which can be easily used to measure the similarity of alternatives and ideal alternatives. Based on the distance measures, the TOPSIS method has been used to rank alternatives, which can reduce the heavy computational burden.

In order to do so, the rest of the paper is organized as follows. In Section 2, some concepts on multi-valued interval neutrosophic sets are reviewed. Some distance measures for multi-valued interval neutrosophic sets are developed. In Section 3, a new multiple attribute decision making method based on DEMATEL and TOPSIS is proposed for multi-valued interval neutrosophic fuzzy information. In Section 4, a numerical example is presented to illustrate the feasibility and practical advantages of the new algorithm. Some conclusions are given in the last section.

\section{Preliminaries}

Definition 1. ([1]) Let $X$ be a universe of discourse, with a generic element in $X$ denoted by $x$. A neutrosophic set $A$ in $X$ is:

$$
A=\left\{<x, T_{A}(x), I_{A}(x), F_{A}(x)>\mid x \in X\right\},
$$

where $T_{A}$ is the truth-membership function, $I_{A}$ is the indeterminacy-membership function and $F_{A}$ is the real falsity-membership function. $T_{A}(x), I_{A}(x)$ and $F_{A}(x)$ are real standard or nonstandard subsets of $] 0^{-}, 1^{+}\left[\right.$. There is no restriction on the sum of $T_{A}(x), I_{A}(x)$ and $F_{A}(x)$, and we can obtain $0^{-} \leq T_{A}(x)+I_{A}(x)+F_{A}(x) \leq 3^{+}$.

Definition 2. ([6]) Let $X$ be a universe of discourse, with a generic element in $X$ denoted by $x$. An interval neutrosophic set (INS) $\hat{A}$ in $X$ is:

$$
A=\left\{<x, \hat{T}_{\hat{A}}(x), \hat{I}_{\hat{A}}(x), \hat{F}_{\hat{A}}(x)>\mid x \in X\right\},
$$

where $\hat{T}_{\hat{A}}$ is the truth-membership function, $\hat{I}_{A}$ is the indeterminacy-membership function and $\hat{F}_{A}$ is the real falsity-membership function. For each point $x$ in $X$, we have $\hat{T}_{\hat{A}}(x)=\left[T_{\hat{A}}^{L}(x), T_{\hat{A}}^{U}(x)\right] \subseteq$ $[0,1], \hat{I}_{\hat{A}}(x)=\left[I_{\hat{A}}^{L}(x), I_{\hat{A}}^{U}(x)\right] \subseteq[0,1], \hat{F}_{A}(x)=\left[F_{\hat{A}}^{L}(x), F_{\hat{A}}^{U}(x)\right] \subseteq[0,1]$ and $\sup \left(T_{\hat{A}}^{U}(x)\right)+\sup \left(I_{\hat{A}}^{U}(x)\right)+$ $\sup \left(F_{\hat{A}}^{U}(x)\right) \leq 3$.

Definition 3. ([27]) Let X be a non-empty finite set. A multi-valued interval neutrosophic fuzzy set (MVINFS) on $X$ is represented by:

$$
\tilde{A}=\left\{<x, \tilde{T}_{\tilde{A}}(x), \tilde{I}_{\tilde{A}}(x), \tilde{F}_{\tilde{A}}(x)>\mid x \in X\right\},
$$

where $\tilde{T}_{\tilde{A}}(x)=\left\{\tilde{\xi} \mid \tilde{\xi} \in \tilde{T}_{\tilde{A}}(x)\right\}, \quad \tilde{I}_{\tilde{A}}(x)=\left\{\tilde{\eta} \mid \tilde{\eta} \in \tilde{I}_{\tilde{A}}(x)\right\}$ and $\tilde{F}_{\tilde{A}}(x)=\left\{\tilde{\gamma} \mid \tilde{\gamma} \in \tilde{F}_{\tilde{A}}(x)\right\}$ are sets of interval values, which are the possible truth membership degrees, indeterminacy-membership degrees and falsity-membership degrees of element $x$ to set $\tilde{A}$ satisfying $\tilde{\xi}=\left[\xi^{L}, \xi^{U}\right] \subseteq$ $[0,1], \quad \tilde{\eta}=\left[\eta^{L}, \eta^{U}\right] \subseteq[0,1], \quad \tilde{\gamma}=\left[\gamma^{L}, \gamma^{U}\right] \subseteq[0,1]$ and $0 \leq \sup \tilde{\xi}^{+}+\sup \tilde{\eta}^{+}+\sup \tilde{\gamma}^{+} \leq 3$, where $\sup \tilde{\xi}^{+}=\bigcup_{\tilde{\xi} \in \tilde{T}_{\tilde{\tilde{A}}}(x)} \max \{\tilde{\xi}\}, \quad \sup \tilde{\eta}^{+}=\bigcup_{\tilde{\eta} \in \tilde{I}_{\tilde{A}}(x)} \max \{\tilde{\eta}\}, \quad \sup \tilde{\gamma}^{+}=\bigcup_{\tilde{\gamma} \in \tilde{F}_{\tilde{A}}(x)} \max \{\tilde{\gamma}\}$. Let $\tilde{a}=<\tilde{T}_{\tilde{A}}(x), \tilde{I}_{\tilde{A}}(x), \tilde{F}_{\tilde{A}}(x)>$ be a multi-valued interval neutrosophic fuzzy element (MVINFE).

Let $\tilde{a}_{1}=<\tilde{T}_{1}, \tilde{I}_{1}, \tilde{F}_{1}>, \tilde{a}_{2}=<\tilde{T}_{2}, \tilde{I}_{2}, \tilde{F}_{2}>$ be two MVINFEs, $k>0,\left[\xi_{1}^{L}, \tilde{\xi}_{1}^{U}\right] \in \tilde{T}_{1},\left[\eta_{1}^{L}, \eta_{1}^{U}\right] \in \tilde{I}_{1}$, $\left[\gamma_{1}^{L}, \gamma_{1}^{U}\right] \in \tilde{F}_{1},\left[\tilde{\xi}_{2}^{L}, \tilde{\xi}_{2}^{U}\right] \in \tilde{T}_{2},\left[\eta_{2}^{L}, \eta_{2}^{U}\right] \in \tilde{I}_{2},\left[\gamma_{2}^{L}, \gamma_{2}^{U}\right] \in \tilde{F}_{2}$. The operational laws of MVINFEs can be defined as follows:

(1) $\tilde{a}_{1} \oplus \tilde{a}_{2}=<\tilde{T}_{1} \oplus \tilde{T}_{2}, \tilde{I}_{1} \otimes \tilde{I}_{2}, \tilde{F}_{1} \otimes \tilde{F}_{2}>=<\cup\left\{\left[\xi_{1}^{L}+\xi_{2}^{L}-\xi_{1}^{L} \xi_{2}^{L}, \xi_{1}^{U}+\xi_{2}^{U}-\xi_{1}^{U} \xi_{2}^{U}\right]\right\}$, $\cup\left\{\left[\eta_{1}^{L} \eta_{2}^{L}, \eta_{1}^{U} \eta_{2}^{U}\right]\right\}, \cup\left\{\left[\gamma_{1}^{L} \gamma_{2}^{L}, \gamma_{1}^{U} \gamma_{2}^{U}\right]\right\}>$, 
(2) $\tilde{a}_{1} \otimes \tilde{a}_{2}=<\tilde{T}_{1} \otimes \tilde{T}_{2}, \tilde{I}_{1} \oplus \tilde{I}_{2}, \tilde{F}_{1} \oplus \tilde{F}_{2}>=<\cup\left\{\left[\xi_{1}^{L} \xi_{2}^{L}, \xi_{1}^{U} \xi_{2}^{U}\right]\right\}, \cup\left\{\left[\eta_{1}^{L}+\eta_{2}^{L}-\eta_{1}^{L} \eta_{2}^{L}, \eta_{1}^{U}+\eta_{2}^{U}-\right.\right.$ $\left.\left.\eta_{1}^{U} \eta_{2}^{U}\right]\right\}, \cup\left\{\left[\gamma_{1}^{L}+\gamma_{2}^{L}-\gamma_{1}^{L} \gamma_{2}^{L}, \gamma_{1}^{U}+\gamma_{2}^{U}-\gamma_{1}^{U} \gamma_{2}^{U}\right]\right\}>$,

(3) $k \tilde{a}_{1}=<\cup\left\{\left[1-\left(1-\xi_{1}^{L}\right)^{k}, 1-\left(1-\xi_{1}^{U}\right)^{k}\right]\right\}, \cup\left\{\left[\left(\eta_{1}^{L}\right)^{k},\left(\eta_{1}^{U}\right)^{k}\right]\right\}, \cup\left\{\left[\left(\gamma_{1}^{L}\right)^{k},\left(\gamma_{1}^{U}\right)^{k}\right]\right\}>$ ，

(4) $\left(\tilde{a}_{1}\right)^{k}=<\cup\left\{\left[\left(\xi_{1}^{L}\right)^{k},\left(\xi_{1}^{U}\right)^{k}\right]\right\}, \cup\left\{\left[1-\left(1-\eta_{1}^{L}\right)^{k}, 1-\left(1-\eta_{1}^{U}\right)^{k}\right]\right\}, \cup\left\{\left[1-\left(1-\gamma_{1}^{L}\right)^{k}, 1-(1-\right.\right.$ $\left.\left.\left.\gamma_{1}^{u}\right)^{k}\right]\right\}>$

Definition 4. ([11]) Let $\tilde{a}_{j}=<\tilde{T}_{j}, \tilde{I}_{j}, \tilde{r}_{j}>$ be MVINFEs and $w=\left(w_{1}, w_{2}, \ldots, w_{n}\right)$ be the weight vector with $w_{i} \geq 0, \sum_{j=1}^{n} w_{j}=1$. The multi-valued interval neutrosophic fuzzy weighted average (MVINFWA) operator can be defined as:

$$
\operatorname{MVINFWA} A_{w}\left(\tilde{a}_{1}, \tilde{a}_{2}, \ldots, \tilde{a}_{n}\right)=w_{1} \tilde{a}_{1}+w_{2} \tilde{a}_{2}+\ldots+w_{n} \tilde{a}_{n} .
$$

By using the operational laws of MVINFEs, we can obtain:

$$
\begin{aligned}
& \operatorname{MVINFWA}_{w}\left(\tilde{a}_{1}, \tilde{a}_{2}, \ldots, \tilde{a}_{n}\right) \\
= & <\left\{\left[1-\prod_{j=1}^{n} \bigcup_{\tilde{\xi}_{j} \in \tilde{T}_{j}}\left(1-\xi_{j}^{L}\right)^{w_{j}}, 1-\prod_{j=1}^{n} \bigcup_{\tilde{\mathcal{S}}_{j} \in \tilde{T}_{j}}\left(1-\xi_{j}^{U}\right)^{w_{j}}\right]\right\},\left\{\left[\prod_{j=1}^{n} \bigcup_{\tilde{\eta}_{j} \in \tilde{I}_{j}}\left(\eta_{j}^{L}\right)^{w_{j}},\right.\right. \\
& \left.\prod_{j=1}^{n} \bigcup_{\tilde{\eta}_{j} \in \tilde{I}_{j}}\left(\eta_{j}^{U}\right)^{w_{j}}\right],\left\{\left[\prod_{j=1}^{n} \bigcup_{\tilde{\gamma}_{j} \in \tilde{F}_{j}}\left(\gamma_{j}^{L}\right)^{w_{j}}, \prod_{j=1}^{n} \bigcup_{\tilde{\gamma}_{j} \in \tilde{F}_{j}}\left(\gamma_{j}^{U}\right)^{w_{j}}\right]\right\}>,
\end{aligned}
$$

where $\tilde{\xi}_{j}=\left[\xi_{j}^{L}, \tilde{\xi}_{j}^{U}\right] \in \tilde{T}_{j}, \tilde{\eta}_{j}=\left[\eta_{j}^{L}, \eta_{j}^{U}\right] \in \tilde{I}_{j}, \tilde{\gamma}_{j}=\left[\gamma_{j}^{L}, \gamma_{j}^{U}\right] \in \tilde{F}_{j}$.

Definition 5. ([27]) For an MVINFE $\tilde{a}=<\tilde{T}_{\tilde{A}}(x), \tilde{I}_{\tilde{A}}(x), \tilde{F}_{\tilde{A}}(x)>$,

$$
s(\tilde{a})=\frac{1}{3}\left(\frac{1}{2 l} \sum_{i=1}^{l}\left(\xi_{i}^{L}+\xi_{i}^{U}\right)+\frac{1}{2 m} \sum_{j=1}^{m}\left(2-\eta_{j}^{L}-\eta_{j}^{U}\right)+\frac{1}{2 n} \sum_{k=1}^{n}\left(2-\gamma_{k}^{L}-\gamma_{k}^{U}\right)\right)
$$

is called the score function of $\tilde{a}$, where $l, m, n$ are the numbers of the interval values in $\tilde{T}_{\tilde{A}}(x), \tilde{I}_{\tilde{A}}(x), \tilde{F}_{\tilde{A}}(x)$, $\tilde{\xi}_{i}=\left[\xi_{i}^{L}, \xi_{i}^{U}\right] \in \tilde{T}_{\tilde{A}}(x), \tilde{\eta}_{i}=\left[\eta_{i}^{L}, \eta_{i}^{U}\right] \in I_{A}(x), \tilde{\gamma}_{k}=\left[\gamma_{k}^{L}, \gamma_{k}^{U}\right] \in \tilde{F}_{\tilde{A}}(x)$, respectively.

Different truth-membership functions, indeterminacy-membership functions and real falsitymembership functions may have different numbers of interval values. In order to define distance more clearly, MVINFEs should be extended until all truth-membership functions, indeterminacy-membership functions and real falsity-membership functions have the same number of interval values according to the risk attitude of decision makers. If the decision makers are risk-seeking, the largest truth-membership, the smallest indeterminacy-membership and the smallest falsity-membership should be added. If decision makers are risk-averse, the smallest truth-membership, the largest indeterminacy-membership and the largest falsity-membership should be added. If decision makers are risk-neutral, the average truth-membership, the average indeterminacy-membership and the average indeterminacy-membership should be added.

The following equation can be used to rank interval numbers.

Definition 6. ([43]) Let $a=\left[a^{L}, a^{U}\right]=\left\{x \mid a^{L} \leq x \leq a^{U}\right\}$. Then, $a$ is an interval number. Let $a=$ $\left[a^{L}, a^{U}\right], b=\left[b^{L}, b^{U}\right]$ be two interval number and $l_{a}=a^{U}-a^{L}, l_{b}=b^{U}-b^{L}$. Then, the possibility of $a>b$ can be defined as:

$$
p(a>b)=\max \left\{1-\max \left\{\frac{b^{U}-a^{L}}{l_{a}+l_{b}}, 0\right\}, 0\right\} .
$$

We generalize the multi-valued neutrosophic fuzzy distance measures to accommodate multi-valued interval neutrosophic fuzzy values and develop the following distance measures.

Definition 7. Let $\tilde{a}_{1}=<\tilde{T}_{1}, \tilde{I}_{1}, \tilde{F}_{1}>$ and $\tilde{a}_{2}=<\tilde{T}_{2}, \tilde{I}_{2}, \tilde{F}_{2}>$ be two MVINFEs, then the normalized Euclidean distance between $\tilde{a}$ and $\tilde{b}$ is defined as follows: 


$$
\begin{aligned}
d(\tilde{a}, \tilde{b})= & \left(\frac { 1 } { 3 } \left(\frac{1}{2 l} \sum_{i=1}^{l}\left(\left(\xi_{1 \sigma(i)}^{L}-\xi_{2 \sigma(i)}^{L}\right)^{2}+\left(\xi_{1 \sigma(i)}^{U}-\xi_{2 \sigma(i)}^{U}\right)^{2}\right)+\frac{1}{2 m} \sum_{j=1}^{m}\left(\left(\eta_{1 \sigma(j)}^{L}-\eta_{2 \sigma(j)}^{L}\right)^{2}+\right.\right.\right. \\
& \left.\left.\left.\left(\eta_{1 \sigma(j)}^{U}-\eta_{2 \sigma(j)}^{U}\right)^{2}\right)+\frac{1}{2 n} \sum_{k=1}^{n}\left(\left(\gamma_{1 \sigma(k)}^{L}-\gamma_{2 \sigma(k)}^{L}\right)^{2}+\left(\gamma_{1 \sigma(k)}^{U}-\gamma_{2 \sigma(k)}^{U}\right)^{2}\right)\right)\right)^{1 / 2}
\end{aligned}
$$

The normalized Hamming distance is defined as:

$$
\begin{aligned}
d(\tilde{a}, \tilde{b})= & \frac{1}{3}\left(\frac{1}{2 l} \sum_{i=1}^{l}\left(\left|\xi_{1 \sigma(i)}^{L}-\xi_{2 \sigma(i)}^{L}\right|+\left|\xi_{1 \sigma(i)}^{U}-\xi_{2 \sigma(i)}^{U}\right|\right)+\frac{1}{2 m} \sum_{j=1}^{m}\left(\left|\eta_{1 \sigma(j)}^{L}-\eta_{2 \sigma(j)}^{L}\right|+\right.\right. \\
& \left.\left.\left|\eta_{1 \sigma(j)}^{U}-\eta_{2 \sigma(j)}^{U}\right|\right)+\frac{1}{2 n} \sum_{k=1}^{u}\left(\left|\gamma_{1 \sigma(k)}^{L}-\gamma_{2 \sigma(k)}^{L}\right|+\left|\gamma_{1 \sigma(k)}^{U}-\gamma_{2 \sigma(k)}^{U}\right|\right)\right)
\end{aligned}
$$

where $\left[\xi_{1 \sigma(k)}^{L}, \xi_{1 \sigma(k)}^{U}\right] \in \tilde{T}_{1},\left[\xi_{2 \sigma(k)}^{L}, \xi_{2 \sigma(k)}^{U}\right] \in \tilde{T}_{2},\left[\eta_{1 \sigma(l)}^{L}, \eta_{1 \sigma(l)}^{U}\right] \in \tilde{I}_{1},\left[\eta_{2 \sigma(l)}^{L}, \eta_{2 \sigma(l)}^{U}\right] \in \tilde{I}_{2},\left[\gamma_{1 \sigma(l)}^{L}, \gamma_{1 \sigma(t)}^{U}\right]$ $\in \tilde{F}_{1},\left[\gamma_{2 \sigma(t)}^{L}, \gamma_{2 \sigma(t)}^{U}\right] \in \tilde{F}_{2}, l, m, n$ are the numbers of interval values in $\tilde{T}_{\tilde{A}}(x), \tilde{I}_{\tilde{A}}(x), \tilde{F}_{\tilde{A}}(x)$, respectively. The distance measures can be proven to satisfy the following properties easily.

(1) $d(\tilde{a}, \tilde{b}) \geq 0$,

(2) $d(\tilde{a}, \tilde{b})=0$, if and only if $\tilde{a}=\tilde{b}$,

(3) $d(\tilde{a}, \tilde{b})=d(\tilde{b}, \tilde{a})$.

\section{The Multi-Valued Interval Neutrosophic Fuzzy Decision Making Method Based on DEMATEL and TOPSIS}

In this section, we develop a new multiple attribute decision making method based on DEMATEL and TOPSIS for multi-valued interval neutrosophic fuzzy information. In this method, DEMATEL has been used to determine attribute weights, and TOPSIS has been used to rank alternatives.

The concrete steps of the DEMATEL are as follows.

Step 1. Generate the direct-relation matrix. Decision makers are asked to assess the effect among factor pairs. The direct-relation matrix is established as $A=\left(a_{i j}\right)_{n \times n}$, where $A$ is an $n \times n$ non-negative matrix, $a_{i j}$ indicates the direct effect of factor $i$ on factor $j$ and the diagonal elements $a_{i i}=0$ for $i=1,2, \ldots, n$.

Step 2. Normalize the direct-relation matrix by $D=A \times S$, where $S=\frac{1}{\max _{1 \leq i \leq n} \sum_{j=1}^{n} a_{i j}}$ and obtain the normalized initial direct-relation matrix as $D=\left(d_{i j}\right)_{n \times n}$.

Step 3. Set up the total-relation matrix by the following equation:

$$
T=\left(t_{i j}\right)_{n \times n}=D(I-D)^{-1},
$$

where $I$ is the $n \times n$ identity matrix and $t_{i j}$ is the indirect effect of criterion $i$ on criterion $j$.

Step 4. Sum each row and each column of matrix $T$ by Equations (10) and (11). The sum of row $i$ is denoted by $r_{i}$ and:

$$
r_{i}=\sum_{j=1}^{n} t_{i j}, i=1,2, \ldots, n
$$

where $r_{i}$ denotes the direct and indirect influence of criterion $i$ on the other criteria and $r_{i}$ is called the the degree of influential impact. The sum of column $j$ is denoted by $c_{j}$ and:

$$
c_{j}=\sum_{i=1}^{n} t_{i j}, j=1,2, \ldots, n,
$$

where $c_{j}$ denotes the direct and indirect effect of criterion $j$ with respect to the other criteria. $r_{i}+c_{i}$ shows all the effects of giving and receiving of criterion $i$. By using the $r_{i}+c_{i}$, the priority weight of each criterion can be calculated as follows:

$$
w_{i}=\frac{r_{i}+c_{i}}{\sum_{i=1}^{n}\left(r_{i}+c_{i}\right)}, i=1,2, \ldots, n
$$

After obtaining the attribute weights, we can rank alternatives by using the TOPSIS method. 
Based on the above analysis, we present the new multiple attribute decision making method for multi-valued interval neutrosophic fuzzy information based on DEMATEL and TOPSIS. For a multiple attribute decision making problem, $\left\{A_{1}, A_{2}, \ldots, A_{m}\right\}$ is the set of alternatives and $\left\{C_{1}, C_{2}, \ldots, C_{n}\right\}$ is the set of attributes. For a complicated decision problem, decision makers would express some extent of hesitation in evaluating alternatives and attributes. In order to model evaluation values more accurately, we use interval neutrosophic fuzzy values, in which the truth-membership function, the indeterminacy-membership function and the real falsity-membership function are all interval values. Many complex decision problems need experts from different fields. Since each expert has his/her own field of expertise, they may be familiar with some attributes and not with other attributes. In order to obtain more accurate evaluation values, they can refuse to give evaluation values or give several evaluation values. Then, we can obtain multi-valued interval neutrosophic fuzzy information. In this case, the new decision making method based on DEMATEL and TOPSIS is developed in the following steps.

\section{Algorithm}

Step 1. Decision makers provide interval neutrosophic fuzzy evaluation values in the pairwise comparison of attributes. Then, the multi-valued interval neutrosophic fuzzy decision matrix is formed as $\tilde{M}=\left(\tilde{m}_{i j}\right)_{n \times n}, \tilde{m}_{i j}=<\tilde{T}_{\tilde{m}_{i j}} \tilde{I}_{\tilde{m}_{i j}}, \tilde{F}_{\tilde{m}_{i j}}>$. $\tilde{T}_{\tilde{m}_{i j}} \tilde{I}_{\tilde{m}_{i j}} \tilde{F}_{\tilde{m}_{i j}}$ are the sets of the truth-membership function, the indeterminacy-membership function and the falsity-membership function, respectively. $\left[\mu_{i j k}^{L}, \mu_{i j k}^{U}\right] \in \tilde{T}_{\tilde{m}_{i j}}\left[v_{i j l}^{L}, v_{i j l}^{U}\right] \in \tilde{I}_{\tilde{m}_{i j}}\left[\kappa_{i j s}^{L}, \kappa_{i j s}^{U}\right] \in \tilde{F}_{\tilde{m}_{i j}}$

$$
\tilde{M}=\left[\begin{array}{lrrr}
0 & \tilde{m}_{12} & \ldots & \tilde{m}_{1 n} \\
\tilde{m}_{21} & 0 & \ldots & \tilde{m}_{2 n} \\
\vdots & \vdots & \ddots & \vdots \\
\tilde{m}_{n 1} & \tilde{m}_{n 2} & \ldots & 0
\end{array}\right]
$$

Step 2. Normalize the initial direct-relation decision matrix. Calculate the average values of the truth-membership, the indeterminacy-membership and the real falsity-membership in each multi-valued interval neutrosophic fuzzy value and transform the multi-valued interval neutrosophic fuzzy decision matrix into single-valued interval neutrosophic fuzzy decision matrix $\tilde{M}^{\prime}=\left(\tilde{m}_{i j}^{\prime}\right)_{n \times n}$ by calculating the average values, where $\tilde{m}_{i j}^{\prime}=<\left[\mu_{i j}^{\prime L}, \mu_{i j}^{\prime U}\right],\left[v^{\prime \prime}{ }_{i j}, v_{i j}^{\prime}{ }_{i j}\right],\left[\kappa_{i j}^{\prime L}, \kappa_{i j}^{\prime \prime}\right]>, \mu_{i j}^{\prime L}=\frac{1}{K_{i j}} \sum_{k=1}^{K_{i j}} \mu_{i j k}^{L}$ $\mu^{\prime}{ }_{i j}^{U}=\frac{1}{K_{i j}} \sum_{k=1}^{K_{i j}} \mu_{i j k}^{U}, \prime^{\prime L}{ }_{i j}^{L}=\frac{1}{L_{i j}} \sum_{l=1}^{L_{i j}} v_{i j l}^{L}, v^{\prime U}{ }_{i j}^{U}=\frac{1}{L_{i j}} \sum_{l=1}^{L_{i j}} v_{i j l}^{U},^{\prime} \kappa_{i j}^{L}=\frac{1}{S_{i j}} \sum_{s=1}^{S_{i j}} \kappa_{i j s}^{L}, \kappa_{i j}^{\prime U}=\frac{1}{S_{i j}} \sum_{s=1}^{S_{i j}} \kappa_{i j s}^{U}$.

$$
\tilde{M}^{\prime}=\left[\begin{array}{lrlr}
0 & \tilde{m}_{12}^{\prime} & \ldots & \tilde{m}_{1 n}^{\prime} \\
\tilde{m}_{21}^{\prime} & 0 & \ldots & \tilde{m}_{2 n}^{\prime} \\
\vdots & \vdots & \ddots & \vdots \\
\tilde{m}_{n 1}^{\prime} & \tilde{m}_{n 2}^{\prime} & \ldots & 0
\end{array}\right]
$$

Normalize decision matrix $\tilde{M}^{\prime}$ into $\tilde{M}^{\prime \prime}=\left(\tilde{m}_{i j}^{\prime \prime}\right)_{n \times n}, \tilde{m}_{i j}^{\prime \prime}=<\left[\mu_{i j}^{\prime \prime}{ }_{i j}, \mu_{i j}^{\prime \prime} U_{i j}\right],\left[v^{\prime \prime}{ }_{i j}^{L}, v^{\prime \prime}{ }_{i j} U_{j}\right],\left[\kappa^{\prime \prime}{ }_{i j}^{L}, \kappa^{\prime \prime}{ }_{i j}^{U}\right]>$,

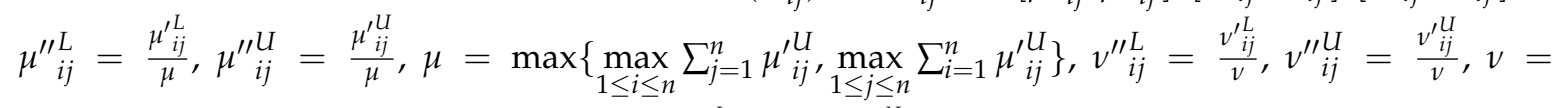

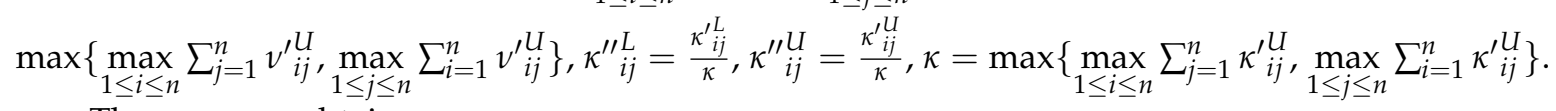

Then, we can obtain: 


$$
\begin{aligned}
& \tilde{M}^{\prime \prime}=\left[\begin{array}{lrrr}
0 & \tilde{m}_{12}^{\prime \prime} & \ldots & \tilde{m}_{1 n}^{\prime \prime} \\
\tilde{m}_{21}^{\prime \prime} & 0 & \ldots & \tilde{m}_{2 n}^{\prime \prime} \\
\vdots & \vdots & \ddots & \vdots \\
\tilde{m}_{n 1}^{\prime \prime} & \tilde{m}_{n 2}^{\prime \prime} & \ldots & 0
\end{array}\right] \\
& \tilde{M}_{\mu^{\prime \prime}{ }_{i j}^{\prime \prime}}^{\prime \prime}=\left[\begin{array}{lrrr}
0 & \mu_{12}^{\prime \prime} U & \ldots & \mu_{1 n}^{\prime \prime} U \\
\mu^{\prime \prime} U & 0 & \ldots & \mu_{2 n}^{\prime \prime} U \\
\vdots & \vdots & \ddots & \vdots \\
\mu_{n 1}^{\prime \prime} U & \mu_{n 2}^{\prime \prime} U & \ldots & 0
\end{array}\right], \\
& \tilde{M}_{v^{\prime \prime}{ }_{i j}^{\prime \prime}}^{\prime \prime}=\left[\begin{array}{lrrr}
0 & v^{\prime \prime}{ }_{12}^{L} & \ldots & v_{1 n}^{\prime \prime L} \\
v_{21}^{\prime \prime L} & 0 & \ldots & v_{2 n}^{\prime \prime L} \\
\vdots & \vdots & \ddots & \vdots \\
v_{n 1}^{\prime \prime L} & v_{n 2}^{\prime \prime L} & \ldots & 0
\end{array}\right], \ldots, \\
& \tilde{M}_{\mathcal{K}^{\prime \prime}{ }_{i j}^{\prime \prime}}^{\prime \prime}=\left[\begin{array}{lrrr}
0 & \kappa^{\prime \prime} U & \ldots & \kappa^{\prime \prime} U \\
\mathcal{K}^{\prime \prime} U & 0 & \ldots & \kappa^{\prime \prime} U \\
21 & 0 & \\
\vdots & \vdots & \ddots & \vdots \\
\kappa^{\prime \prime}{ }_{n 1} U & \kappa^{\prime \prime}{ }_{n 2} U & \ldots & 0
\end{array}\right] .
\end{aligned}
$$

Step 3. Calculate the total-relation matrix, which can be calculated by using the above six crisp matrices and can be represented as follows.

$$
\tilde{M}^{\prime \prime \prime}=\left[\begin{array}{lrlr}
0 & \tilde{m}_{12}^{\prime \prime \prime} & \ldots & \tilde{m}_{1 n}^{\prime \prime \prime} \\
\tilde{m}_{21}^{\prime \prime \prime} & 0 & \ldots & \tilde{m}_{2 n}^{\prime \prime \prime} \\
\vdots & \vdots & \ddots & \vdots \\
\tilde{m}_{n 1}^{\prime \prime \prime} & \tilde{m}_{n 2}^{\prime \prime \prime} & \ldots & 0
\end{array}\right],
$$

where $\tilde{m}_{i j}^{\prime \prime \prime}=<\left[\mu_{i j}^{\prime \prime \prime}{ }_{i j}^{L}, \mu^{\prime \prime \prime}{ }_{i j}\right],\left[v^{\prime \prime \prime}{ }_{i j}^{L}, v_{i j}^{\prime \prime \prime}{ }_{i j}\right], \kappa^{\prime \prime \prime \prime}{ }_{i j}^{L}, \kappa^{\prime \prime \prime}{ }_{i j}^{U}>$. The elements in total-relation matrix can be calculated by the following equation $\left[\mu^{\prime \prime \prime}{ }_{i j}^{L}\right]=\tilde{M}_{\mu^{\prime \prime}{ }_{i j}^{\prime \prime}}^{\prime \prime} \times\left(I-\tilde{M}_{\mu^{\prime \prime}{ }_{i j}^{L}}^{\prime \prime}\right)^{-1},\left[\mu^{\prime \prime \prime}{ }_{i j} U_{i j}\right]=\tilde{M}_{\mu^{\prime \prime}}^{\prime \prime} \times\left(I-\tilde{M}_{\mu^{\prime \prime}}^{\prime \prime}{ }_{i j}^{\prime \prime}\right)^{-1}$,

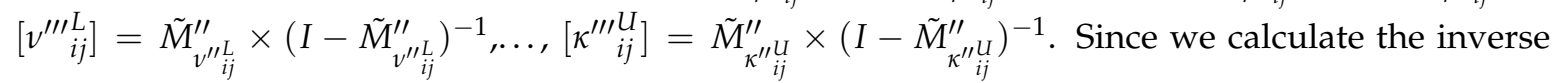
matrices of matrices $I-\tilde{M}_{\mu^{\prime \prime}{ }_{i j}^{\prime \prime}}^{\prime \prime}, \ldots, I-\tilde{M}_{\mathcal{K}^{\prime \prime}{ }_{i j}^{\prime}}^{\prime \prime}$ in calculating the total-relation matrix, the elements in the total-relation matrix are generalized interval neutrosophic fuzzy values.

Step 4. Determine the prominence and influence of each attribute. Calculate the sum $\hat{r}_{i}$ of rows and sum $\hat{c}_{i}$ of columns by the following equations.

$$
\begin{aligned}
& \hat{r}_{i}=\sum_{j=1}^{n} m_{i j}^{\prime \prime \prime}(i=1,2, \ldots, n), \\
& \hat{c}_{j}=\sum_{i=1}^{n} m_{i j}^{\prime \prime \prime}(j=1,2, \ldots, n) .
\end{aligned}
$$

Step 5. Calculate the weights of attributes by the following equation:

$$
w_{i}=\frac{\sqrt{\left(S\left(\hat{r}_{i}+\hat{c}_{i}\right)\right)^{2}+\left(S\left(\hat{r}_{i}-\hat{c}_{i}\right)\right)^{2}}}{\sum_{i=1}^{n} \sqrt{\left(S\left(\hat{r}_{i}+\hat{c}_{i}\right)\right)^{2}+\left(S\left(\hat{r}_{i}-\hat{c}_{i}\right)\right)^{2}}}, i=1,2, \ldots, n .
$$

Step 6. Decision makers evaluate alternatives with respect to attributes with interval neutrosophic fuzzy values, and then multi-valued interval neutrosophic fuzzy decision matrix can be obtained as 
$\tilde{D}=\left(\tilde{a}_{i j}\right)_{m \times n}$, where $\tilde{a}_{i j}=<\tilde{T}_{\tilde{a}_{i j}}, \tilde{a}_{\tilde{a}_{i j}}, \tilde{F}_{\tilde{a}_{i j}}>$. Extend the decision matrix according to the risk attitude of decision makers until all the multi-valued interval neutrosophic fuzzy values have the same number of truth-memberships, indeterminacy-memberships and real falsity-memberships. Then, the extended decision matrix can be obtained as $\tilde{D}^{\prime}=\left(\tilde{a}_{i j}^{\prime}\right)_{m \times n}, \tilde{a}_{i j}^{\prime}=<\tilde{T}_{\tilde{a}_{i j}^{\prime}}^{\prime} \tilde{I}_{\tilde{a}_{i j}^{\prime}}^{\prime} \tilde{F}_{\tilde{a}_{i j}^{\prime}}^{\prime}>$.

Step 7. Calculate the weighted decision matrix $\tilde{D}^{\prime \prime}=\left(\tilde{a}_{i j}^{\prime \prime}\right)_{m \times n}$ by using the attribute weights obtained from Equation (11), where $\tilde{a}_{i j}^{\prime \prime}=w_{j} \tilde{a}_{i j}^{\prime}$.

Step 8. Determine the multi-valued interval neutrosophic fuzzy positive ideal solution (MVINFPIS), denoted as $\tilde{I}^{+}$and the multi-valued interval neutrosophic fuzzy negative ideal solution (MVINFNIS), denoted as $\tilde{I}^{-}$, as follows:

$$
\begin{aligned}
& \tilde{I}^{+}=\left(\tilde{a}_{1}^{+}, \tilde{a}_{2}^{+}, \ldots, \tilde{a}_{n}^{+}\right)=\left(\max _{i}\left\{\tilde{a}_{i 1}^{\prime \prime}\right\}, \max _{i}\left\{\tilde{a}_{i 2}^{\prime \prime}\right\}, \ldots, \max _{i}\left\{\tilde{a}_{i n}^{\prime \prime}\right\}\right), \\
& \tilde{I}^{-}=\left(\tilde{a}_{1}^{-}, \tilde{a}_{2}^{-}, \ldots, \tilde{a}_{n}^{-}\right)=\left(\min _{i}\left\{\tilde{a}_{i 1}^{\prime \prime}\right\}, \min _{i}\left\{\tilde{a}_{i 2}^{\prime \prime}\right\}, \ldots, \min _{i}\left\{\tilde{a}_{i n}^{\prime \prime}\right\}\right) .
\end{aligned}
$$

Step 9. Calculate the distances of each alternative's weighted evaluation values to the MVINFPIS and MVINFNIS:

$$
\begin{aligned}
& d_{i}^{+}=\sum_{j=1}^{n} d\left(\tilde{a}_{i j}^{\prime \prime}, \tilde{a}_{j}^{+}\right), i=1,2, \ldots m, \\
& d_{i}^{-}=\sum_{j=1}^{n} d\left(\tilde{a}_{i j}^{\prime \prime}, \tilde{a}_{j}^{-}\right), i=1,2, \ldots, m .
\end{aligned}
$$

Step 10. Determine the closeness coefficients $C C_{i}$ of alternatives and rank alternatives according to the closeness coefficients.

$$
C C_{i}=\frac{d_{i}^{-}}{d_{i}^{-}+d_{i}^{+}}, i=1,2, \ldots, m .
$$

The new algorithm has the following advantages. First, evaluation values are in the form of multi-valued interval neutrosophic fuzzy values, in which the hesitation of decision makers and fuzzy nature of human thinking can be reflected accurately. Second, the DEMATEL method has been used to determine attribute weights, which can overcome the existing shortcomings of attribute-determining methods, especially for large-scale, complex decision problems such as supply chain management, strategic management, etc. Third, the TOPSIS method has been used to rank alternatives to avoid the complexity of computation and affect the decision makers' subjectiveness.

\section{Numerical Example}

In this section, we apply the new algorithm to the problem of truck selection (adapted from [44]) in the logistics industry to illustrate its feasibility and effectiveness. The logistics industry has witnessed a high speed growth with the development of e-commerce, mobile e-commence, etc. Land transportation plays an important role in logistics, which is generally operated in complex environments. Truck selection is important to logistics companies. Suppose that a logistics company wants to purchase a number of trucks. Experts from different departments are invited to form a committee to evaluate and select trucks. The following four attributes are considered: $C_{1}$-security and technology, $C_{2}$-economy, $C_{3}$-comfort and aesthetics, $C_{4}$-maintenance. After pre-evaluation, there are still five types of trucks for further selection $A_{i}(i=1,2, \ldots, 5)$. The new method has been used to rank alternatives, concretely.

Step 1. Decision makers compare attributes pairwise by using multi-valued interval neutrosophic fuzzy values, and decision matrix $\tilde{M}=\left(\tilde{m}_{i j}\right)_{4 \times 4}$ is formed as in Table 1 .

Step 2. Transform decision matrix $\tilde{M}$ into single-valued interval neutrosophic fuzzy decision matrix $\tilde{M}^{\prime}$ as in Table 2 by calculating the average values of the truth-membership, the indeterminacy-membership and the falsity-membership. Normalize decision matrix $\tilde{M}^{\prime}$ to obtain 


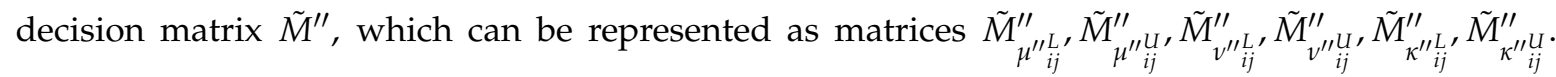
For example, since $\mu=\max \left\{\max _{1 \leq i \leq 4} \sum_{j=1}^{4} \mu_{i j}^{\prime U} \max _{1 \leq j \leq 4} \sum_{i=1}^{4} \mu_{i j}^{\prime U}\right\}=2$ and $\mu^{\prime \prime}{ }_{i j}^{L}=\frac{\mu^{\prime L}}{\mu}$, then decision matrix $\tilde{M}_{\mu^{\prime \prime}{ }_{i j}^{L}}^{\prime \prime}$ can be obtained.

$$
\begin{aligned}
\tilde{M}_{\mu^{\prime \prime}{ }_{i j}^{\prime \prime}}^{\prime \prime}= & {\left[\begin{array}{lccr}
0 & 0.2262 & 0.1259 & 0.3 \\
0.3 & 0 & 0.2 & 0.35 \\
0.2764 & 0.25 & 0 & 0.1760 \\
0.1760 & 0.15 & 0.3 & 0
\end{array}\right], } \\
\tilde{M}_{\mu^{\prime \prime}{ }_{i j}^{\prime \prime}}^{\prime \prime}= & {\left[\begin{array}{lccr}
0 & 0.2764 & 0.1760 & 0.3500 \\
0.3500 & 0 & 0.2500 & 0.4000 \\
0.3118 & 0.3000 & 0 & 0.2262 \\
0.2262 & 0.2000 & 0.3500 & 0
\end{array}\right], \ldots, } \\
\tilde{M}_{\mathcal{K}^{\prime \prime}{ }_{i j}^{\prime \prime}}^{\prime \prime}= & {\left[\begin{array}{lccr}
0 & 0.2261 & 0.3252 & 0.2941 \\
0.2941 & 0 & 0.3529 & 0.3529 \\
0.2353 & 0.2353 & 0 & 0.2941 \\
0.3529 & 0.2661 & 0.2353 & 0
\end{array}\right], }
\end{aligned}
$$

Table 1. Decision matrix $\tilde{M}$.

\begin{tabular}{lll}
\hline & $C_{1}$ & $C_{2}$ \\
\hline$C_{1}$ & 0 & $\langle\{[0.5,0.6],[0.4,0.5]\},\{[0.2,0.3]\},\{[0.3,0.4],[0.4,0.5]\}\rangle$ \\
$C_{2}$ & $\langle\{[0.6,0.7]\},\{[0.4,0.5]\},\{[0.4,0.5]\}\rangle$ & 0 \\
$C_{3}$ & $\langle\{[0.5,0.6],[0.6,0.7]\},\{[0.5,0.6]\},\{[0.3,0.4]\}\rangle$ & $\langle\{[0.5,0.6]\},\{[0.2,0.3]\},\{[0.3,0.4]\}\rangle$ \\
$C_{4}$ & $\langle\{[0.3,0.4],[0.4,0.5]\},\{[0.6,0.7]\},\{[0.5,0.6]\}\rangle$ & $\langle\{0.3,0.4]\},\{[0.3,0.4]\},\{[0.3,0.4],[0.4,0.5]\}\rangle$ \\
\hline & $C_{3}$ & $C_{4}$ \\
\hline$C_{1}$ & $\langle\{[0.2,0.3],[0.3,0.4]\},\{[0.1,0.2]\},\{[0.4,0.5],[0.5,0.6]\}\rangle$ & $\langle\{[0.6,0.7]\},\{[0.3,0.4]\},\{[0.4,0.5]\}\rangle$ \\
$C_{2}$ & $\langle\{[0.4,0.5]\},\{[0.5,0.6]\},\{[0.3,0.4],[0.4,0.5]\}\rangle$ & $\langle\{[0.7,0.8]\},\{[0.5,0.6]\},\{[0.6,0.7]\}\rangle$ \\
$C_{3}$ & 0 & $\langle\{[0.3,0.4],[0.4,0.5]\},\{[0.5,0.6]\},\{[0.4,0.5]\}\rangle$ \\
$C_{4}$ & $\langle\{[0.6,0.7]\},\{[0.4,0.5],[0.5,0.6]\},\{[0.3,0.4]\}\rangle$ & 0 \\
\hline
\end{tabular}

Table 2. Decision matrix $\tilde{M}^{\prime}$.

\begin{tabular}{lll}
\hline & $C_{1}$ & $C_{2}$ \\
\hline$C_{1}$ & 0 & $\langle\{[0.4523,0.5528]\},\{[0.2000,0.3000]\},\{[0.3519,0.4523]\}\rangle$ \\
$C_{2}$ & $\langle\{[0.6000,0.7000]\},\{[0.4000,0.5000]\},\{[0.4000,0.5000]\}\rangle$ & 0 \\
$C_{3}$ & $\langle\{[0.5528,0.6536]\},\{[0.5000,0.6000]\},\{[0.3000,0.4000]\}\rangle$ & $\langle\{[0.5000,0.6000]\},\{[0.2000,0.3000]\},\{[0.3000,0.4000]\}\rangle$ \\
$C_{4}$ & $\langle\{[0.3519,0.4523]\},\{[0.6000,0.7000]\},\{[0.5000,0.6000]\}\rangle$ & $\langle\{[0.3000,0.4000]\},\{[0.3000,0.4000]\},\{[0.3519,0.4523]\}\rangle$ \\
\hline & $C_{3}$ & $C_{4}$ \\
\hline$C_{1}$ & $\langle\{[0.2517,0.3519]\},\{[0.1000,0.2000]\},\{[0.4523,0.5528]\}\rangle$ & $\langle\{[0.6000,0.7000]\},\{[0.3000,0.4000]\},\{[0.4000,0.5000]\}\rangle$ \\
$C_{2}$ & $\langle\{[0.4000,0.5000]\},\{[0.5000,0.6000]\},\{[0.3519,0.4523]\}\rangle$ & $\langle\{[0.7000,0.8000]\},\{[0.5000,0.6000]\},\{[0.6000,0.7000]\}\rangle$ \\
$C_{3}$ & 0 & $\langle\{[0.3519,0.4523]\},\{[0.5000,0.6000]\},\{[0.4000,0.5000]\}\rangle$ \\
$C_{4}$ & $\langle\{[0.6000,0.7000]\},\{[0.4523,0.5528]\},\{[0.3000,0.4000]\}\rangle$ & 0 \\
\hline
\end{tabular}

Step 3. Calculate the total-relation matrix $\tilde{M}^{\prime \prime \prime}=\left(\tilde{m}_{i j}^{\prime \prime \prime}\right)$ by using decision matrices

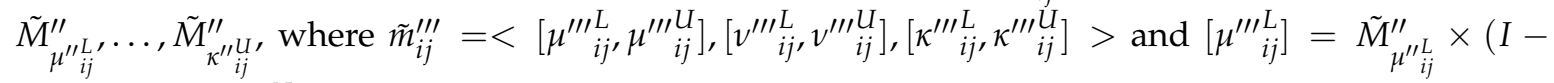
$\left.\tilde{M}_{\mu^{\prime \prime}{ }_{i j}^{L}}^{\prime \prime}\right)^{-1}, \ldots,\left[\mathcal{K}^{\prime \prime \prime}{ }_{i j}\right]=\tilde{M}_{\mathcal{K}^{\prime \prime}{ }_{i j}^{\prime \prime}}^{\prime \prime} \times\left(I-\tilde{M}_{\kappa^{\prime \prime}{ }_{i j}^{\prime \prime}}^{\prime \prime}\right)^{-1}$. The results are shown in Table 3.

Step 4. Calculate the average value of each row to obtain $\hat{r}_{i}$ and the average of each column to obtain $\hat{c}_{i}$ by using Equations (13) and (14). The results are as follows:

$\hat{r}_{1}=<\{[0.5578,1.3476]\},\{[0.2354,0.6184]\},\{[0.5763,1.7225]\}>, \hat{r}_{2}=<\{[0.6841,1.5973]\}$, $\{[0.4862,1.0403]\},\{[0.6525,1.8953]\}>, \hat{r}_{3}=<\{[0.5935,1.4968]\},\{[0.4113,0.9152]\},\{[0.4961$, 
$1.5389]\}>, \hat{r}_{4}=<\{[0.5345,1.3104]\},\{[0.4507,0.9823]\},\{[0.5616,1.6879]\}>, \hat{c}_{1}=<\{[0.6164$, $1.4502]\},\{[0.5148,1.0887]\},\{[0.5794,1.7281]\}>, \hat{c}_{2}=<\{[0.5326,1.3083]\},\{[0.6164,1.4502]\}$, $\{[0.4995,1.5472]\}>, \hat{c}_{3}=<\{[0.5476,1.4191]\},\{[0.6164,1.4502]\},\{[0.5936,1.7616]\}>, \hat{c}_{4}=<$ $\{[0.6733,1.5747]\},\{[0.4408,0.9649]\},\{[0.6141,1.8076]\}>$.

Table 3. Decision matrix $\tilde{M}^{\prime \prime \prime}$.

\begin{tabular}{ll}
\hline & $C_{1}$ \\
\hline$C_{1}$ & $<\{[0.4330,1.2162]\},\{[0.2093,0.6268]\},\{[0.4389,1.5669]\}>$ \\
$C_{2}$ & $<\{[0.7688,1.7019]\},\{[0.6125,1.2709]\},\{[0.6885,1.9523]\}>$ \\
$C_{3} \quad<\{[0.6894,1.5117]\},\{[0.5852,1.1798]\},\{[0.5306,1.5928]\}>$ \\
$C_{4} \quad<\{[0.5743,1.3708]\},\{[0.6522,1.2774]\},\{[0.6594,1.8002]\}>$ \\
\hline & $C_{2}$ \\
\hline$C_{1}$ & $<\{[0.5578,1.3127]\},\{[0.2100,0.5311]\},\{[0.5502,0.6134]\}>$ \\
$C_{2}$ & $<\{[0.4641,1.3004]\},\{[0.2238,0.6509]\},\{[0.4290,1.5442]\}>$ \\
$C_{3}$ & $<\{[0.6083,1.3799]\},\{[0.2907,0.7167]\},\{[0.4768,1.4449]\}>$ \\
$C_{4}$ & $<\{[0.5003,1.2400]\},\{[0.3471,0.7935]\},\{[0.5418,1.5864]\}>$ \\
\hline & $C_{3}$ \\
\hline$C_{1}$ & $<\{[0.5063,1.2744]\},\{[0.2052,0.5828]\},\{[0.6604,1.8433]\}>$ \\
$C_{2}$ & $<\{[0.6467,1.5260]\},\{[0.5215,1.0551]\},\{[0.7369,2.0188]\}>$ \\
$C_{3}$ & $<\{[0.4087,1.5260]\},\{[0.2450,0.6976]\},\{[0.3858,1.4259]\}>$ \\
$C_{4} \quad<\{[0.6087,1.3496]\},\{[0.4682,0.9824]\},\{[0.5914,1.7585]\}>$ \\
\hline & $C_{4}$ \\
\hline$C_{1}$ & $<\{[0.7142,1.5870],\{[0.3169,0.7327]\},\{[0.6558,1.8664]\}>$ \\
$C_{2}$ & $<\{[0.8569,1.8610],\{[0.5870,1.1843]\},\{[0.7557,2.0658]\}>$ \\
$C_{3}$ & $<\{[0.6676,1.5697],\{[0.5242,1.0668]\},\{[0.5912,1.6918]\}>$ \\
$C_{4}$ & $<\{[0.4545,1.2810],\{[0.3352,0.8757]\},\{[0.4536,1.6064]\}>$ \\
\hline
\end{tabular}

Step 5. Calculate the weights of attributes by using Equation (15) to obtain $w_{1}=0.2160, w_{2}=0.2808, w_{3}=0.2682, w_{4}=0.2350$.

Step 6. Decision makers evaluate alternatives with respect to attributes by using the multi-valued interval neutrosophic fuzzy values. Then, decision matrix $\tilde{D}=\left(\tilde{a}_{i j}\right)_{5 \times 4}$ is formed as in Table 4 .

Table 4. Decision matrix $\tilde{D}$.

\begin{tabular}{lll}
\hline Alternative & $\boldsymbol{C}_{\mathbf{1}}$ & $C_{\mathbf{2}}$ \\
\hline$A_{1}$ & $<\{[0.4,0.5],[0.5,0.6]\},\{[0.3,0.4]\},\{[0.3,0.4]$, & $<\{[0.2,0.3],[0.3,0.4]\},\{[0.1,0.2]\},\{[0.4,0.5]$, \\
& $[0.4,0.5]\}>$ & $[0.5,0.6]\}>$ \\
$A_{2}$ & $<\{[0.6,0.7]\},\{[0.1,0.2]\},\{[0.2,0.3]\}>$ & $<\{[0.6,0.7]\},\{[0.1,0.2]\},\{[0.1,0.2]\}>$ \\
$A_{3}$ & $<\{[0.5,0.6]\},\{[0.2,0.3]\},\{[0.3,0.4]\}>$ & $<\{[0.5,0.6]\},\{[0.2,0.3]\},\{[0.2,0.3]\}>$ \\
$A_{4}$ & $<\{[0.6,0.7],[0.7,0.8]\},\{[0.1,0.2]\},\{[0.2,0.3]\}>$ & $<\{[0.3,0.4]\},\{[0.3,0.4]\},\{[0.1,0.2],[0.3,0.4]\}>$ \\
$A_{5}$ & $<\{[0.8,0.9]\},\{[0.3,0.4]\},\{[0.1,0.2]\}>$ & $<\{[0.4,0.5]\},\{[0.4,0.5]\},\{[0.5,0.6]\}>$ \\
\hline & $C_{3}$ & $C_{4}$ \\
\hline$A_{1}$ & $<\{[0.4,0.5],[0.5,0.6]\},\{[0.1,0.2]\},\{[0.3,0.4]\}>$ & $<\{[0.7,0.8],[0.8,0.9]\},\{[0.1,0.2]\},\{[0.2,0.3]\}>$ \\
$A_{2}$ & $<\{[0.3,0.4]\},\{[0.1,0.2]\},\{[0.1,0.2],[0.2,0.3]\}>$ & $<\{[0.3,0.4]\},\{[0.3,0.4],[0.4,0.5]\},\{[0.4,0.5]$, \\
& & \\
$A_{3}$ & $<\{[0.3,0.4],[0.5,0.7]\},\{[0.2,0.3]\},\{[0.2,0.3]\}>$ & $<\{[0.8,0.9]\},\{[0.5,0.6]\},\{[0.3,0.4]\}>$ \\
$A_{4}$ & $<\{[0.7,0.8]\},\{[0.1,0.2]\},\{[0.1,0.2]\}>$ & $<\{[0.3,0.5]\},\{[0.2,0.3],[0.3,0.5]\},\{[0.5,0.6]\}>$ \\
$A_{5}$ & $<\{[0.2,0.3]\},\{[0.4,0.5]\},\{[0.3,0.4]\}>$ & $<\{[0.4,0.5]\},\{[0.1,0.2],[0.2,0.3]\},\{[0.2,0.3]\}>$ \\
\hline
\end{tabular}

Step 7. Extend the decision matrix according to the risk attitude of decision makers. Assume that decision makers are risk-averse, then add the smallest interval truth-membership, the largest interval indeterminacy-membership and the largest real interval falsity-membership. The extended decision matrix $\tilde{D}^{\prime}=\left(\tilde{a}_{i j}^{\prime}\right)_{5 \times 4}$ is omitted due to space limitations. 
Step 8. Calculate the extended weighted decision matrix $\tilde{D}^{\prime \prime}=\left(\tilde{a}_{i j}^{\prime \prime}\right)_{5 \times 4}, \tilde{a}_{i j}^{\prime \prime}=w_{j} \tilde{a}_{i j}^{\prime}$. The results are shown in Table 5.

Table 5. Extended weighted decision matrix $\tilde{D}^{\prime \prime}$.

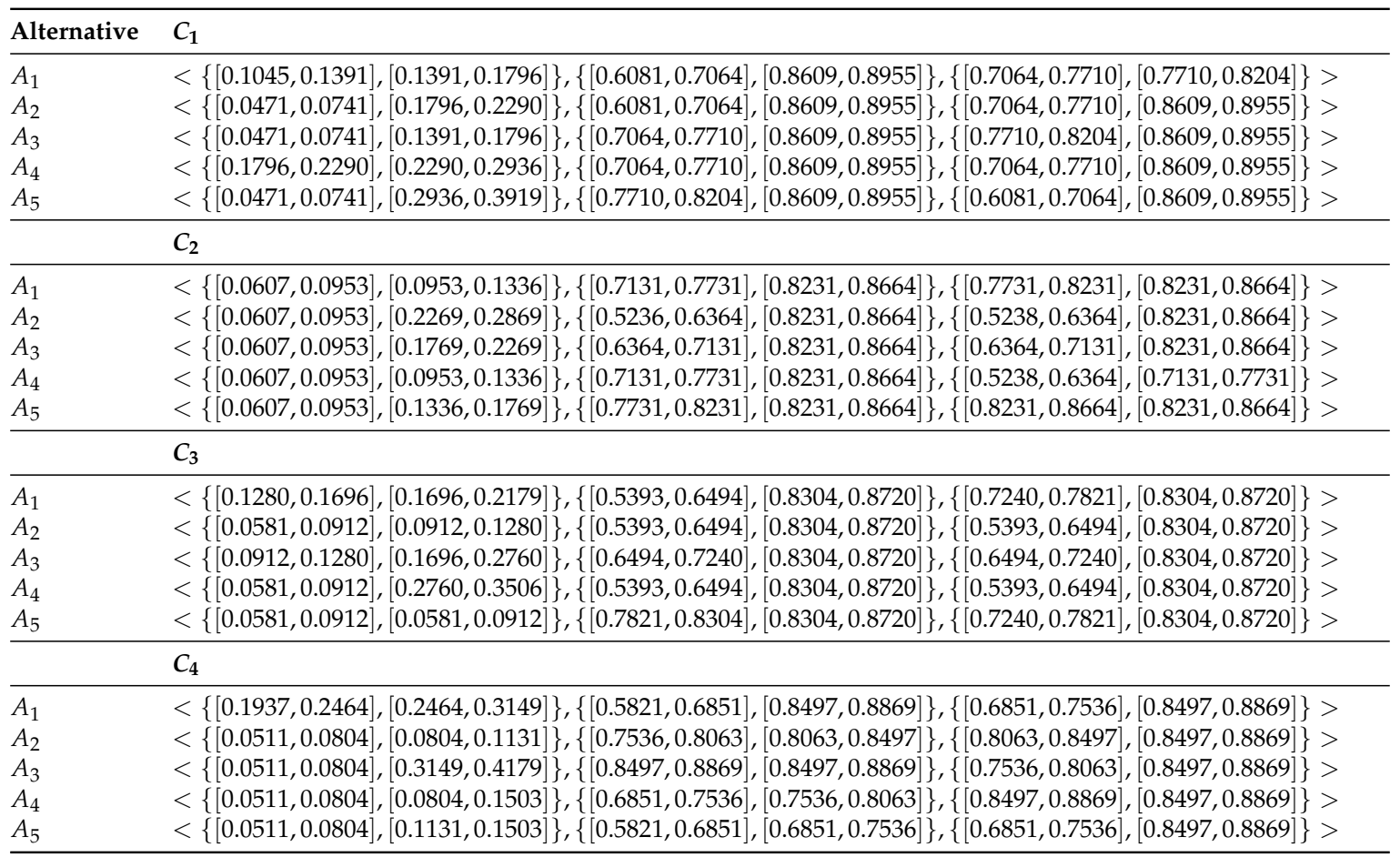

Step 9. MVINFPIS and MVINFNIS can be determined by using Equations (16) and (17):

MVINFPIS: $(<\{[0.1796,0.2290],[0.2290,0.2936]\},\{[0.7064,0.7710],[0.8609,0.8955]\},\{[0.7064$, $0.7710],[0.8609,0.8955]\}><\{[0.0607,0.0953],[0.2269,0.2869]\},\{[0.5236,0.6364],[0.8231,0.8664]\}$, $\{[0.5238,0.6364],[0.8231,0.8664]\}>,<\{[0.0581,0.0912],[0.2760,0.3506]\},\{[0.5393,0.6494],[0.8304$, $0.8720]\},\{[0.5393,0.6494],[0.8304,0.8720]\}>,<\{[0.1937,0.2464],[0.2464,0.3149]\},\{[0.5821,0.6851]$, $[0.8497,0.8869]\},\{[0.6851,0.7536],[0.8497,0.8869]\}>)$.

MVINFNIS: $(<\{[0.0471,0.0741],[0.1391,0.1796]\},\{[0.7064,0.7710],[0.8609,0.8955]\},\{[0.7710$, $0.8204],[0.8609,0.8955]\}>,<\{[0.0607,0.0953],[0.1336,0.1769]\},\{[0.7731,0.8231],[0.8231,0.8664]\}$, $\{[0.8231,0.8664],[0.8231,0.8664]\}>,<\{[0.0581,0.0912],[0.0581,0.0912]\},\{[0.7821,0.8304],[0.8304$, $0.8720]\},\{[0.7240,0.7821],[0.8304,0.8720]\}>,<\{[0.0511,0.0804],[0.0804,0.1131]\},\{[0.7536,0.8063]$, $[0.8063,0.8497]\},\{[0.8063,0.8497],[0.8497,0.8869]\}>)$.

The distances of each alternative's evaluation values to the MVINFPIS and MVINFNIS can be calculated by using Equations (18) and (19) as

$$
d_{1}^{+}=0.4046, d_{2}^{+}=0.4302, d_{3}^{+}=0.4625, d_{4}^{+}=0.3116, d_{5}^{+}=0.6796, d_{1}^{-}=0.4560,
$$
$d_{2}^{-}=0.4550, d_{3}^{-}=0.4143, d_{4}^{-}=0.5374, d_{5}^{-}=0.2640$.

Step 10. The closeness coefficients can be calculated by using Equation (20) as

$C C_{1}=0.5298, C C_{2}=0.5140, C C_{3}=0.4725, C C_{4}=0.6330, C C_{5}=0.2798$.

The alternatives can be ranked accordingly as $A_{4} \succ A_{1} \succ A_{2} \succ A_{3} \succ A_{5}$, and the optimal alternative is $A_{4}$.

In order to illustrate the advantages of the new method, we compare it with some other methods. If evaluation values are given in crisp numbers, the multi-valued interval neutrosophic fuzzy decision matrix reduces to multi-valued neutrosophic fuzzy decision matrix $M$ as in Table 6 (denoted as Method 1). Then, we transform the multi-valued neutrosophic fuzzy decision matrix into the single-value neutrosophic fuzzy decision matrix by calculating the average values 
and normalizing. Calculate the total-relation matrix $M^{\prime}=\left(m_{i j}^{\prime}\right)_{4 \times 4}$ as in Table 7, where $m_{i j}^{\prime}=<\left\{\mu_{i j}^{\prime}\right\},\left\{v_{i j}^{\prime}\right\},\left\{\kappa_{i j}^{\prime}\right\}>,\left[\mu_{i j}^{\prime}\right]=M_{\mu_{i j}} \times\left(I-M_{\mu_{i j}}\right)^{-1},\left[v_{i j}^{\prime}\right]=M_{v_{i j}} \times\left(I-M_{v_{i j}}\right)^{-1},\left[\kappa_{i j}^{\prime}\right]=$ $M_{\kappa_{i j}} \times\left(I-M_{\kappa_{i j}}\right)^{-1}$. The elements in the total-relation matrix $M^{\prime}$ are generalized neutrosophic fuzzy values. The average value of each rank can be calculated as $r_{i}^{\prime}$, and the average value of each column can be calculated as $c_{i}^{\prime}$. Concretely, $r_{1}^{\prime}=<\{1.2342\},\{0.8826\},\{1.4407\}>$, $r_{2}^{\prime}=<\{1.0721\},\{0.4676\},\{1.0452\}>, r_{3}^{\prime}=<\{1.0943\},\{0.6177\},\{1.1303\}>, r_{4}^{\prime}=<\{0.7549\}$, $\{0.6298\},\{0.6211\}>, c_{1}^{\prime}=<\{0.6422\},\{0.6897\},\{0.6842\}>, c_{2}^{\prime}=<\{0.5827\},\{0.4344\},\{0.5510\}>$, $c_{3}^{\prime}=<\{0.5916\},\{0.5409\},\{0.5829\}>, c_{4}^{\prime}=<\{0.6791\},\{0.6211\},\{0.6528\}>$. Let $w_{i}=$ $\frac{\sqrt{\left(S\left(r_{i}^{\prime}+c_{i}^{\prime}\right)\right)^{2}+\left(S\left(r_{i}^{\prime}-c_{i}^{\prime}\right)\right)^{2}}}{\sum_{i=1}^{n} \sqrt{\left(S\left(r_{i}^{\prime}+c_{i}^{\prime}\right)\right)^{2}+\left(S\left(r_{i}^{\prime}-c_{i}^{\prime}\right)\right)^{2}}}$. Attribute weights can be calculated as $w_{1}=0.2815, w_{2}=0.2124$, $w_{3}=0.2260, w_{4}=0.2802$. Decision makers evaluate alternatives with multi-valued neutrosophic fuzzy values, and the decision matrix is formed as in Table 8. Assume decision makers are risk-averse. Then, we extend the decision matrix by adding the smallest truth-membership, the largest indeterminacy-membership and the largest real falsity-membership. Then, the extended decision matrix is obtained. Calculate the weighted decision matrix by using attribute weights and results are shown in Table 9. Determine the multi-valued neutrosophic fuzzy positive ideal solution (MNFPIS) $\mathrm{m}^{+}$and the multi-valued neutrosophic fuzzy negative ideal solution (MNFNIS) $\mathrm{m}^{-}$as $m^{+}=\left(m_{1}^{+}, m_{2}^{+}, m_{3}^{+}, m_{4}^{+}\right)=\left(\max _{i} m_{i 1}, \max _{i} m_{i 2}, \max _{i} m_{i 3}, \max _{i} m_{i 4}\right)=(<\{0.2274,0.2875\},\{0.5230$, $0.8227\},\{0.6357,0.8227\}>,<\{0.0463,0.1769\},\{0.6132,0.8631\},\{0.6132,0.8631\}>,<\{0.0492$, $0.0774\},\{0.5943,0.8550\},\{0.5943,0.6951\}>,<\{0.2863,0.3630\},\{0.5246,0.8235\},\{0.6370,0.8235\}>)$, $m^{-}=\left(m_{1}^{-}, m_{2}^{-}, m_{3}^{-}, m_{4}^{-}\right)=\left(\min _{i} m_{i 1}, \min _{i} m_{i 2}, \min _{i} m_{i 3}, \min _{i} m_{i 4}\right)=(<\{0.1339,0.1773\},\{0.7125$, $0.8227\},\{0.7125,0.7726\}>,<\{0.0463,0.1028\},\{0.8231,0.8631\},\{0.8631,0.8631\}>,<\{0.0492$, $0.0492\},\{0.8130,0.8550\},\{0.7618,0.8550\}>,<\{0.0606,0.0951\},\{0.6370,0.8235\},\{0.8235,0.8235\}>)$. The distances of each weighted evaluation value to the MNFPIS $a^{+}$and MNFNIS $a^{-}$can be calculated as $d_{1}^{\prime+}=0.3503, d_{2}^{\prime+}=0.3450, d_{3}^{\prime+}=0.3999, d_{4}^{\prime+}=0.2975, d_{5}^{\prime+}=0.5589, d_{1}^{\prime-}=0.4030$, $d_{2}^{\prime-}=0.3608, d_{3}^{\prime-}=0.2873, d_{4}^{\prime-}=0.3607, d_{5}^{\prime-}=0.2747$. The closeness coefficients can be calculated as $C C_{1}^{\prime}=0.5350, C C_{2}^{\prime}=0.5112, C C_{3}^{\prime}=0.4181, C C_{4}^{\prime}=0.5480, C C_{5}^{\prime}=0.3296$. The alternatives can be ranked as $A_{4} \succ A_{1} \succ A_{2} \succ A_{3} \succ A_{5}$, and the optimal alternative is $A_{4}$. From the result, we can obtain similar ranking results. If evaluation values are in the form of multi-valued neutrosophic fuzzy values, the attribute weights are different from those of the interval forms. The attributes $C_{1}$ and $C_{4}$ have relatively big weights, while in the multi-valued interval neutrosophic fuzzy situation, the attributes $C_{2}$ and $C_{3}$ have relatively big weights. Since interval values are more appropriate to model fuzzy and uncertain information, more accurate decision results can be obtained by using interval multi-valued neutrosophic fuzzy values.

We use the MVINFWA operator to aggregate multi-valued interval neutrosophic fuzzy values into collective ones, and the attribute weights are also taken as $(0.2160,0.2808,0.2682,0.2350)$, which are the same as those of the proposed method (denoted as Method 2). The first six steps are the same as the proposed method. Then, we use the MVINFWA operator aggregate evaluation values (the results have been omitted due to space limitations). The scores of the collective evaluation values can be calculated as $S\left(A_{1}\right)=0.6605, S\left(A_{2}\right)=0.6991, S\left(A_{3}\right)=0.6834, S\left(A_{4}\right)=0.7016, S\left(A_{5}\right)=0.6324$. Compare $S\left(A_{i}\right)(i=1,2, \ldots, 5)$. We can obtain $A_{4} \succ A_{2} \succ A_{3} \succ A_{1} \succ A_{5}$. The ranking results are similar to the proposed method. $A_{2}$ and $A_{3}$ have different rankings in the two methods.

Table 6. Decision matrix $M$.

\begin{tabular}{lllll}
\hline & $C_{1}$ & $C_{2}$ & $C_{3}$ & $C_{4}$ \\
\hline$C_{1}$ & 0 & $\langle\{0.5,0.4\},\{0.2\},\{0.3,0.4\}\rangle$ & $\langle\{0.2,0.3\},\{0.1\},\{0.4,0.5\}\rangle$ & $\langle\{0.6\},\{0.3\},\{0.4\}\rangle$ \\
$C_{2}$ & $\langle\{0.6\},\{0.4\},\{0.4\}\rangle$ & 0 & $\langle\{0.4\},\{0.5\},\{0.3,0.4\}\rangle$ & $\langle\{0.7\},\{0.5\},\{0.6\}\rangle$ \\
$C_{3}$ & $\langle\{0.5,0.6\},\{0.5\},\{0.3\}\rangle$ & $\langle\{0.5\},\{0.2\},\{0.3\}\rangle$ & 0 & $\langle\{0.3,0.4\},\{0.5\},\{0.4\}\rangle$ \\
$C_{4}$ & $\langle\{0.3,0.4\},\{0.6\},\{0.5\}\rangle$ & $\langle\{0.3\},\{0.3\},\{0.3,0.4\}\rangle$ & $\langle\{0.6\},\{0.4,0.5\},\{0.3\}\rangle$ & 0 \\
\hline
\end{tabular}


Table 7. Total-relation matrix $M^{\prime}$.

\begin{tabular}{lll}
\hline & $C_{1}$ & $C_{2}$ \\
\hline$C_{1}<\{0.9927\},\{0.4213\},\{1.2141\}>$ & $<\{1.0715\},\{0.3422\},\{1.0696\}>$ \\
$C_{2}<\{1.4835\},\{1.0615\},\{1.5491\}>$ & $<\{1.0610\},\{0.4505\},\{0.9694\}>$ \\
$C_{3}<\{1.3203\},\{0.9732\},\{1.4587\}>$ & $<\{1.1606\},\{0.5000\},\{1.0434\}>$ \\
$C_{4}<\{1.1403\},\{1.0742\},\{1.5408\}>$ & $<\{0.9952\},\{0.5777\},\{1.0985\}>$ \\
\hline & $C_{3}$ & $C_{4}$ \\
\hline$C_{1}<\{1.0261\},\{0.3657\},\{1.1854\}>$ & $<\{1.3569\},\{0.5202\},\{1.3345\}>$ \\
$C_{2}<\{1.2826\},\{0.8494\},\{1.2351\}>$ & $<\{1.6377\},\{0.96788\},\{1.5303\}>$ \\
$C_{3}<\{0.9437\},\{0.4902\},\{0.9466\}>$ & $<\{1.3462\},\{0.8580\},\{1.3264\}>$ \\
$C_{4}<\{1.1247\},\{0.7655\},\{1.1539\}>$ & $<\{1.0450\},\{0.6625\},\{1.1581\}>$ \\
\hline
\end{tabular}

Table 8. Decision matrix $D$.

\begin{tabular}{lll}
\hline Alternative & $C_{\boldsymbol{1}}$ & $C_{\mathbf{2}}$ \\
\hline$A_{1}$ & $<\{0.4,0.5\},\{0.3\},\{0.3,0.4\}>$ & $<\{0.2,0.3\},\{0.1\},\{0.4,0.5\}>$ \\
$A_{2}$ & $<\{0.6\},\{0.1\},\{0.2\}>$ & $<\{0.6\},\{0.1\},\{0.1\}>$ \\
$A_{3}$ & $<\{0.5\},\{0.2\},\{0.3\}>$ & $<\{0.5\},\{0.2\},\{0.2\}>$ \\
$A_{4}$ & $<\{0.6,0.7\},\{0.1\},\{0.2\}>$ & $<\{0.3\},\{0.3\},\{0.1,0.3\}>$ \\
$A_{5}$ & $<\{0.8\},\{0.3\},\{0.1\}>$ & $<\{0.4\},\{0.4\},\{0.5\}>$ \\
\hline & $C_{3}$ & $C_{4}$ \\
\hline$A_{1}$ & $<\{0.4,0.5\},\{0.1\},\{0.3\}>$ & $<\{0.7,0.8\},\{0.1\},\{0.2\}>$ \\
$A_{2}$ & $<\{0.3\},\{0.1\},\{0.1,0.2\}>$ & $<\{0.3\},\{0.3,0.4\},\{0.4,0.5\}>$ \\
$A_{3}$ & $<\{0.3,0.5\},\{0.2\},\{0.2\}>$ & $<\{0.8\},\{0.5\},\{0.3\}>$ \\
$A_{4}$ & $<\{0.7\},\{0.1\},\{0.1\}>$ & $<\{0.3\},\{0.2,0.3\},\{0.5\}>$ \\
$A_{5}$ & $<\{0.2\},\{0.4\},\{0.3\}>$ & $<\{0.4\},\{0.1,0.2\},\{0.2\}>$ \\
\hline
\end{tabular}

Table 9. The weighted decision matrix $D^{\prime}$.

\begin{tabular}{lll}
\hline Alternative & $C_{1}$ & $C_{2}$ \\
\hline$A_{1}$ & $<\{0.1339,0.1773\},\{0.7125,0.8227\},\{0.7125,0.7726\}>$ & $<\{0.0463,0.0730\},\{0.6132,0.8631\},\{0.8231,0.8631\}>$ \\
$A_{2}$ & $<\{0.0609,0.2274\},\{0.5230,0.8227\},\{0.6357,0.8227\}>$ & $<\{0.0463,0.1769\},\{0.6132,0.8631\},\{0.6132,0.8631\}>$ \\
$A_{3}$ & $<\{0.0609,0.1773\},\{0.6357,0.8227\},\{0.7125,0.8227\}>$ & $<\{0.0463,0.1369\},\{0.7105,0.8631\},\{0.7105,0.7744\}>$ \\
$A_{4}$ & $<\{0.2274,0.2875\},\{0.5230,0.8227\},\{0.6357,0.8227\}>$ & $<\{0.0463,0.0730\},\{0.7744,0.8631\},\{0.6132,0.8632\}>$ \\
$A_{5}$ & $<\{0.0609,0.3643\},\{0.7125,0.8227\},\{0.5230,0.8227\}>$ & $<\{0.0463,0.1028\},\{0.8231,0.8631\},\{0.8631,0.8631\}>$ \\
\hline & $C_{3}$ & $C_{4}$ \\
\hline$A_{1}$ & $<\{0.1090,0.1450\},\{0.5943,0.8550\},\{0.7618,0.8550\}>$ & $<\{0.2863,0.3630\},\{0.5246,0.8235\},\{0.6370,0.8235\}>$ \\
$A_{2}$ & $<\{0.0492,0.0774\},\{0.5943,0.8550\},\{0.5943,0.6951\}>$ & $<\{0.0606,0.0951\},\{0.7131,0.7736\},\{0.7736,0.8235\}>$ \\
$A_{3}$ & $<\{0.0774,0.1450\},\{0.6951,0.8550\},\{0.6951,0.8550\}>$ & $<\{0.0606,0.3630\},\{0.8235,0.8235\},\{0.7137,0.8235\}>$ \\
$A_{4}$ & $<\{0.0492,0.2382\},\{0.5943,0.8550\},\{0.5943,0.8550\}>$ & $<\{0.0606,0.0951\},\{0.6370,0.8235\},\{0.8235,0.8235\}>$ \\
$A_{5}$ & $<\{0.0492,0.0492\},\{0.8130,0.8550\},\{0.7618,0.8550\}>$ & $<\{0.0606,0.1334\},\{0.5246,0.8235\},\{0.6370,0.8235\}>$ \\
\hline
\end{tabular}

We summarize the above analysis in Table 10. In the proposed method, the fuzzy and uncertain information is modeled by using multi-valued interval neutrosophic fuzzy values. The attribute weights are determined by using DEMATEL, which can model the interactions and dependencies among attributes. The TOPSIS method is used to rank alternatives, and the amount of computation has been reduced greatly.

Table 10. The comparisons of the characteristic of different methods.

\begin{tabular}{cccc}
\hline Method & $\begin{array}{c}\text { Information by Multi-Valued } \\
\text { Interval Neutrosophic Fuzzy Value }\end{array}$ & $\begin{array}{c}\text { Whether the Weights Are } \\
\text { Determined by DEMATEL }\end{array}$ & $\begin{array}{c}\text { Whether Alternatives Are } \\
\text { Ranked by TOPSIS }\end{array}$ \\
\hline Method 1 & No & Yes & No \\
Method 2 & Yes & No & No \\
Our proposed method & Yes & Yes & Yes \\
\hline
\end{tabular}


We further compare the proposed method with some other existing methods $[29,45]$. We first transform the multi-valued interval neutrosophic fuzzy values into interval neutrosophic fuzzy values by using the interval-valued neutrosophic fuzzy averaging operator. Then, we use the interval-valued neutrosophic fuzzy weighted averaging operator [45] or TOPSIS [29] to obtain the ranking results, which are shown in Table 11. The attribute weights are taken as $(0.2160,0.2808,0.2682,0.2350)$, which are the same as our proposed method, in order to facilitate comparison. From the results, we can see that, though the optimal alternative is the same, the rankings of alternatives are different. Moreover, the attribute weights are not determined properly in the existing methods. Hence, compared to other methods, the proposed method can determine attribute weights reasonably and solve complicated decision problems by using multi-valued interval neutrosophic fuzzy values.

Table 11. Comparisons of different methods.

\begin{tabular}{lcc}
\hline Method & Alternatives Ranking & Optimal Alternative \\
\hline Zhao et al. [45] & $A_{4} \succ A_{2} \succ A_{3} \succ A_{1} \succ A_{5}$ & $A_{4}$ \\
Chi and Liu [29] & $A_{4} \succ A_{2} \succ A_{3} \succ A_{1} \succ A_{5}$ & $A_{4}$ \\
Our proposed method & $A_{4} \succ A_{1} \succ A_{2} \succ A_{3} \succ A_{5}$ & $A_{4}$ \\
\hline
\end{tabular}

\section{Conclusions}

In this study, a new multiple attribute decision making method is developed based on the DEMATEL and TOPSIS methods for multi-valued interval neutrosophic fuzzy information. The interactions of attributes have been modeled by DEMATEL, and the computational complexity has decreased by using the TOPSIS method to rank alternatives. The main advantages of the new method are as follows: the evaluation values are given as multi-valued interval neutrosophic fuzzy values, which can be used to model fuzziness and hesitation since each element has several interval truth-memberships, interval indeterminacy-memberships and interval real falsity-memberships. Decision makers can give more than one evaluation value if they are familiar with the attributes and can refuse to give evaluation values if they are not familiar with the attributes, which can reduce the effect of unreasonable evaluation values due to decision makers. In most classical multiple attribute decision making methods, attributes are assumed to be independent of each other. However, various interactions and interdependencies exist among attributes. The new method can model dependencies among attributes by using the DEMATEL method. Though the computational cost increases by using the multi-valued interval neutrosophic fuzzy values, the new model is still a polynomial-time algorithm and can be solved easily. Moreover, the amount of computation been has reduced greatly by using the TOPSIS method.

The proposed method can be extended in the following direction: The proposed method has been used to solve a relative small-scale problem in this paper due to the space limitations. In fact, the proposed method can be used to solve more complicated, large-scale decision problems such as global supplier selection, emergency management, etc. Other ranking methods, such as ELECTRE, VIKOR and PROMETHEE, can also be used to rank alternatives instead of TOPSIS. Other tools to model fuzzy information can also be used, including fuzzy values, intuitionistic fuzzy values, linguistic arguments, etc.

Acknowledgments: The authors thank anonymous referees for their helpful remarks. This work is partly supported by the National Natural Science Foundation of China (No. 11401457), the Postdoctoral Science Foundation of China (2015M582624) and the Shaanxi Province Postdoctoral Science Foundation of China.

Author Contributions: Wei Yang conceived the idea and wrote the paper; Yongfeng Pang revised the paper. The authors have read and approved the final manuscript.

Conflicts of Interest: The authors declare that there is no conflict of interests regarding the publication of this paper. 


\section{References}

1. Smarandache, F. A Unifying Field in Logics. Neutrosophy: Neutrosophic Probability, Set and Logic; American Research Press: Rehoboth, DE, USA, 1998.

2. Zadeh, L.A. Fuzzy sets. Inf. Control 1965, 8, 338-353.

3. Gorzalczany, M.B. A method of inference in approximate reasoning based on interval-valued fuzzy sets. Fuzzy Sets Syst. 1987, 21, 1-17.

4. Atanassov, K. Intuitionistic fuzzy sets. Fuzzy Sets Syst. 1986, 20, 87-96.

5. Torra, V. Hesitant fuzzy sets. Int. J. Intell. Syst. 2010, 25, 529-539.

6. Wang, H.; Smarandache, F.; Zhang Y.; Sunderraman, R. Single valued neutrosophic sets. In Proceedings of the 10th International Conference on Fuzzy Theory and Technology, Salt Lake City, UT, USA, 21-26 July 2005.

7. Guo, Y.H.; Şengür, A. A novel image segmentation algorithm based on neutrosophic similarity clustering. Appl. Soft Comput. 2014, 25, 391-398.

8. Maji, P.K. An application of weighted neutrosophic soft sets in a decision-making problem. Facet. Uncertain. Appl. 2015, 125, 215-223.

9. Kazim, H.; Fatih, T.M. Segmentation of SAR images using improved artificial bee colony algorithm and neutrosophic set. Appl. Soft Comput. 2014, 21, 433-443.

10. Chen, J.Q.; Ye, J.; Du, S.G. Vector Similarity Measures between Refined Simplified Neutrosophic Sets and Their Multiple Attribute Decision-Making Method. Symmetry 2017, 9, 153, doi:10.3390/sym9080153.

11. Wu, X.H.; Wang, J.Q.; Peng, J.J.; Chen, X.H. Cross-Entropy and Prioritized Aggregation Operator with Simplified Neutrosophic Sets and Their Application in Multi-Criteria Decision-Making Problems. Int. J. Fuzzy Syst. 2016, 18, 1104-1116.

12. Jiang, W.; Shou, Y.H. A Novel Single-Valued Neutrosophic Set Similarity Measure and Its Application in Multicriteria Decision-Making. Symmetry 2017, 9, 12, doi:10.3390/sym9080127.

13. Wang, H.; Smarandache, F.; Zhang Y.Q.; Sunderraman, R. Single valued neutrosophic sets. Multispace Multistruct. 2010, 4, 410-413.

14. Ye, J. Multicriteria decision-making method using the correlation coefficient under single-valued neutrosophic environment. Int. J. Gen. Syst. 2013, 42, 386-394.

15. Ye, J. Single valued neutrosophic cross-entropy for multicriteria decision making problems. Appl. Math. Model. 2014, 38, 1170-1175.

16. Yang, H.L.; Guo, Z.L.; She, Y.H.; Liao, X.W. On single-valued neutrosophic relations. J. Intell. Fuzzy Syst. 2016, 30, 1045-1056.

17. Biswas, P.; Pramanik S.; Giri, B.C. TOPSIS method for multi-attribute group decision-making under single-valued neutrosophic environment. Neural Comput. Appl. 2016, 27, 727-737.

18. Majumdar, P.; Samant, S.K. On similarity and entropy of neutrosophic sets. J. Intell. Fuzzy Syst. 2014, $26,1245-1252$.

19. Liu, P.D.; Chu, Y.C.; Li, Y.W.; Chen, Y.B. Some generalized neutrosophic number hamacher aggregation operators and their application to group decision making. J. Intell. Fuzzy Syst. 2014, 16, 242-255.

20. Wang, J.Q.; Li, X.E. TODIM method with multi-valued neutrosophic sets. Control Dec. 2015, 30, 1139-1142.

21. Maji, P.K. Neutrosophic Soft set. Ann. Fuzzy Math. Inf. 2013, 5, 157-168.

22. Ye, J. Trapezoidal neutrosophic set and its application to multiple attribute decision-making. Neural Comput. Appl. 2015, 26, 1157-1166.

23. Ye, J. An extended TOPSIS method for multiple attribute group decision making based on single-valued neutrosophic linguistic numbers. J. Intell. Fuzzy Syst. 2015, 28, 247-255.

24. Wang, H.; Smarandache, F.; Zhang, Y.Q. Interval Neutrosophic Sets and Logic: Theory and Applications in Computing; Hexis: Phoenix, AZ, USA, 2005.

25. Rdvan, A. Cross-entropy measure on interval neutrosophic sets and its applications in multicriteria decision making. Neural Comput. Appl. 2017, 28, 1177-1187.

26. Liu, P.D.; Tang, G.L. Some power generalized aggregation operators based on the interval neutrosophic sets and their application to decision making. J. Intell. Fuzzy Syst. 2016, 30, 2517-2528.

27. Liu, P.D.; Shi, L.L. The generalized hybrid weighted average operator based on interval neutrosophic hesitant set and its application to multiple attribute decision making. Neural Comput. Appl. 2015, 26, 457-471. 
28. Yang, W.; Shi, J.R.; Pang, Y.F.; Zheng, X.Y. Linear assignment method for interval neutrosophic sets. Neural Comput. Appl. 2018, 29, 553-564.

29. Chi, P.P.; Liu, P.D. An extended TOPSIS method for multiple attribute decision making problems based on interval neutrosophic set. Neural Comput. Appl. 2013, 1, 63-70.

30. Gabus, A.; Fontela, E. World Problems an Invitation to Further Thought within the Framework of DEMATEL; Battelle Geneva Research Centre: Geneva, Switzerland, 1972.

31. Stević, Ž.; Pamučar, D.; Vasiljević, M.; Stojić, G.; Korica, S. Novel Integrated Multi-Criteria Model for Supplier Selection: Case Study Construction Company. Symmetry 2017, 9, 279, doi:10.3390/sym9110279.

32. Lin, C.J.; Wu, W.W. A causal analytical method for group decision making under fuzzy environment. Expert Syst. Appl. 2008, 34, 205-213.

33. Jassbi, J.; Mohamadnejad, F.; Nasrollahzadeh, H. A Fuzzy DEMATEL framework for modeling cause and effect relationships of strategy map. Expert Syst. Appl. 2011, 38, 5967-5973.

34. Nikjoo, A.V.; Saeedpoor, M. An intuitionistic fuzzy DEMATEL methodology for prioritising the components of SWOT matrix in the Iranian insurance industry. Int. J. Oper. Res. 2014, 20, 439-452.

35. Govindan, K.; Khodaverdi, R.; Vafadarnikjoo, A. Intuitionistic fuzzy based DEMATEL method for developing green practices and performances in a green supply chain. Expert Syst. Appl. 2015, 42, 7207-7220.

36. Hosseini, M.B.; Tarokh, M.J. Type-2 fuzzy set extension of DEMATEL method combined with perceptual computing for decision making. J. Ind. Eng. Int. 2013, 9, 1-10.

37. Abdullah, L.; Zulkifli, N. Integration of fuzzy AHP and interval type-2 fuzzy DEMATEL: An application to human resource management. Expert Syst. Appl. 2015, 42, 4397-4409.

38. Hwang, C.L.; Yoon, K. Multiple Attribute Decision Making, Methods and Applications: A State-of-the-Art Survey; Springer: New York, NY, USA, 1981.

39. Ren, F.L.; Kong, M.M.; Zheng, P. A New Hesitant Fuzzy Linguistic TOPSIS Method for Group Multi-Criteria Linguistic Decision Making. Symmetry 2017, 9, 289, doi:10.3390/sym9120289.

40. Chen, Z.P.; Yang, W. An MAGDM based on constrained FAHP and FTOPSIS and its application to supplier selection. Math. Comput. Model. 2011, 54, 2802-2815.

41. Yang, W.; Chen, Z.P.; Zhang, F. New Group Decision Making Method in Intuitionistic Fuzzy Setting Based on TOPSIS. Technol. Econ. Dev. Econ. 2017, 23, 441-461.

42. Yang, W.; Pang, Y.F.; Shi, J.R.; Wang, C.J. Linguistic hesitant intuitionistic fuzzy decision-making method based on VIKOR. Neural Comput. Appl. 2018, 29, 613-626.

43. Chen, N.; Xu, Z.S.; Xia, M.M. Interval-valued hesitant preference relations and their applications to group decision making. Know-Based Syst. 2013, 37, 528-540.

44. Baykasoğlu, A.; Kaplanoğlu, V.; Durmusğlu, Z.D.U.; Şahin, A. Integrating fuzzy DEMATEL and fuzzy hierarchical TOPSIS methods for truck selection. Expert Syst. Appl. 2013, 40, 899-907.

45. Zhao, A.W.; Du, J.G.; Guan, H.J. Interval valued neutrosophic sets and multi-attribute decision-making based on generalized weighted aggregation operator. J. Intell. Fuzzy Syst. 2015, 29, 2697-2706.

(C) 2018 by the authors. Licensee MDPI, Basel, Switzerland. This article is an open access article distributed under the terms and conditions of the Creative Commons Attribution (CC BY) license (http:// creativecommons.org/licenses/by/4.0/). 